\title{
Hiponatremia aguda grave en un paciente esquizofrénico potomano
}

\section{Severe and acute hyponatremia in a schizophrenic patient with potomania}

\author{
M.D. Macías Robles', M.P. López Fonticiella', C. Maciá Bobes², A. Fernández San Martín
}

\section{RESUMEN}

La hiponatremia aguda grave ocasiona una encefalopatía metabólica, cuya fisiopatología es el edema cerebral, y los síntomas más severos son las convulsiones y el coma. Se presenta el caso de una hiponatremia extrema de origen multifactorial en un paciente esquizofrénico potomano. La potomanía no suele ocasionar hiponatremia, salvo coexistencia con otros mecanismos desencadenantes. El paciente descrito presentaba datos de secreción inadecuada de vasopresina (SIADH) y una hipokaliemia intensa, secundaria a vómitos y al tratamiento con indapamida, que perpetúa el déficit extracelular de sodio. En el tratamiento del paciente, el único fármaco de introducción reciente fue el aripiprazol, neuroléptico con el que se han notificado casos de secreción inadecuada de vasopresina. La corrección de la hiponatremia aguda grave es una urgencia vital, independientemente de la causa, y consiste en la administración de suero salino al $3 \%$. El sodio no debe aumentar en más de $25 \mathrm{mmol} / \mathrm{L}$ durante las primeras 24-48 horas para evitar un daño cerebral secundario.

Palabras clave. Convulsiones. Esquizofrenia. Hiponatremia. Aripiprazol. Potomanía.

\begin{abstract}
Acute and severe hyponatremia causes a metabolic encephalothy. It is physiopathologically based on the cerebral edema, and its fatal symptoms include seizures and coma. We present a case of an extreme hyponatremia of multifactorial etiology in a schizophrenic patient with potomania. Potomania does not usually cause hyponatremia, unless it coexists with other trigger factors. This patient had a syndrome of inappropriate antidiuretic hormone (SIADH), and a deep hypokaliemia, due to vomiting and a treatment with indapamida, which perpetuates the deficit of extracellular sodium. In the patient's treatment, aripiprazole was the only recently introduced drug with which cases of inappropriate vasopressin secretion have been reported. Management of a severe hyponatremia must be considered a vital emergency, independent of the cause, and 3\% hypertonic saline must be administered. The increase of the sodium level must not be higher than $25 \mathrm{mmol} / \mathrm{L}$ in the first $24-48$ hours, to avoid a secondary brain injury.
\end{abstract}

Key words. Seizures. Schizophrenia. Hyponatremia. Aripiprazole. Potomania.
1. Servicio de Urgencias del Hospital San Agustín, Avilés (Asturias)

2. Sección de Endocrinología del Hospital San Agustín, Avilés (Asturias)

Recepción el 11 de diciembre de 2008

Aceptación provisional el 22 de enero de 2009

Aceptación definitiva el 28 de enero de 2009

\section{Correspondencia:}

$\mathrm{M}^{\mathrm{a}}$ Dolores Macías Robles

Avda. San Agustín, 16-3ㅜ B

33400 Avilés. Asturias

Tfno. 985527292

Correo electrónico: mdmacias@telefonica.net 


\section{INTRODUCCIÓN}

La vasopresina u hormona antidiurética $(\mathrm{ADH})$ es secretada por la neurohipófisis; su acción fisiológica es reducir la excreción de agua al promover la concentración de la orina. La secreción deficiente de la ADH puede ser primaria o secundaria. La forma primaria suele deberse a la agenesia o a la destrucción irreversible de la neurohipófisis y se denomina diabetes insípida central. El déficit secundario de $\mathrm{ADH}$ se debe a la inhibición de su secreción por una ingesta excesiva de líquidos y recibe el nombre de polidipsia primaria, que se divide en tres grupos:

1. Polidipsia dipsógena o dipsomanía en la que se produce un aumento inapropiado de la sed debido a una alteración del mecanismo osmorregulador que se traduce en la necesidad brusca, inmotivada e imperiosa de beber grandes cantidades de líquido. Se relaciona con trastornos orgánicos cerebrales, fármacos, o, más frecuentemente, es idiopática.

2. Polidipsia psicógena o potomanía, que no se acompaña de sed y la polidipsia se debe al deseo frecuente de beber grandes cantidades de líquido, asociado a una actitud placentera. Se presenta como parte de un trastorno psiquiátrico.

3. Polidipsia iatrogénica que tiene su origen en las recomendaciones de los profesionales de la salud o de medios populares para aumentar la ingesta de líquidos para prevenir o mejorar otros trastor$\operatorname{nos}^{1}$.

La polidipsia psicógena está presente hasta en el $20 \%$ de los pacientes psiquiátricos crónicos; en muchos de estos pacientes coexisten alteraciones que van a conllevar un exceso de ADH o que son causa reconocida de SIADH que pueden producir desequilibrios hidroelectrolíticos severos, ocasionar una encefalopatía metabólica y poner en peligro su vida ${ }^{1,2}$.

Presentamos el caso de un varón esquizofrénico potomano con una hiponatremia aguda grave y crisis convulsivas. Se discuten los mecanismos patogénicos implicados, medidas terapéuticas y profilácticas.

\section{CASO CLÍNICO}

Varón de 42 años con antecedentes de tabaquismo, hipertensión arterial, esquizofrenia paranoide crónica e historia de ingesta de al menos 10 litros de agua al día desde hacía años. Acudió al Servicio de Urgencias por disminución progresiva del nivel de conciencia de 2 días de evolución tras la supuesta toma de 10 comprimidos de clorazepato dipotásico de $50 \mathrm{mg}$, que se siguió de vómitos después de la ingesta. Su tratamiento era: aripiprazol, clorazepato dipotásico, flufenazina, biperideno e indapamida. En la exploración física estaba afebril, TA 110/70 mmHg, la saturación de oxígeno $96 \%$, consciente, letárgico, obnubilado, la auscultación cardiopulmonar era normal y no presentaba edemas en miembros inferiores. A los 30 minutos de su llegada al Servicio de urgencias presentó empeoramiento de su nivel de conciencia con Glasgow de 7 y una convulsión tónico-clónica generalizada que revirtió con $2 \mathrm{mg}$ de clonazepan. Dada su situación neurológica se procedió a intubación orotraqueal. En la bioquímica de sangre destacó: glucosa $134 \mathrm{mg} / \mathrm{dl}$, urea $12 \mathrm{mg} / \mathrm{dl}$, creatinina $0,42 \mathrm{mg} / \mathrm{dl}$, sodio $98 \mathrm{mmol} / \mathrm{L}$ (133-145), potasio $1,67 \mathrm{mmol} / \mathrm{L}(3,3-5,10)$, osmolalidad $200 \mathrm{mOsm} / \mathrm{kg}$ (275-295). En la gasometría se demostró una alcalosis metabólica ( $\mathrm{pH} 7,55$ y bicarbonato $37,7 \mathrm{mmol} / \mathrm{L}$ ). El hemograma y la coagulación eran normales; y en la orina se encontró: sodio $10 \mathrm{mmol} / \mathrm{L}$ (30-300), potasio $28 \mathrm{mmol} / \mathrm{L}$ (25-125) y osmolalidad $270 \mathrm{mOsm} / \mathrm{kg}$ (300-1090). La radiografía de tórax y la TAC craneal fueron normales. En el electrocardiograma se objetivó ritmo sinusal, PR 0,30 segundos y aplanamiento de la onda T. A la vista de los resultados analíticos y el empeoramiento neurológico, el paciente se trasladó a la Unidad de Cuidados Intensivos donde se iniciaron medidas de soporte respiratorio con ventilación mecánica, aporte de sodio, potasio y restricción hídrica. El sodio se repuso con suero salino al 3\%, aumentando en las primeras 8 horas a $110 \mathrm{mmol} / \mathrm{L}$, con lo que mejoró el nivel de conciencia. En las siguientes 36 horas se recuperaron progresivamente las cifras normales de sodio y potasio. No presentó complicaciones posteriores.

\section{DISCUSIÓN}

Entre los diagnósticos psiquiátricos de la polidipsia psicógena, la esquizofrenia explica el $83 \%$ de los casos; también puede asociarse a anorexia y bulimia nerviosas, trastornos afectivos, autismo, abuso de alcohol y trastornos de la personalidad. Existe historia de ingesta excesiva de agua en el $50-80 \%$ de estos pacientes y, generalmente, se describe la polidipsia a los 5-15 años del inicio de la enfermedad psiquiátrica ${ }^{2}$. 
En la polidipsia psicógena la excesiva ingesta de líquidos aumenta el agua corporal, reduciendo la osmolalidad plasmática, la secreción de vasopresina y la concentración de la orina, lo cual provoca un aumento compensador de la eliminación de agua urinaria libre que varia en proporción directa a la ingesta. Esto hace difícil una sobrehidratación clínicamente importante, a menos que algún fármaco o trastorno que estimule la acción de la vasopresina interfiera con la diuresis compensadora ${ }^{1}$.

Cuando el impulso para consumir agua sobrepasa la capacidad de eliminación del riñón (12 litros al día), el incremento primario de agua puede llegar a producir una hiponatremia hipotónica con descenso de la osmolalidad del plasma. En la hiponatremia de los pacientes psiquiátricos con polidipsia se han descrito además otros mecanismos implicados. Estos pacientes pueden desarrollar hiponatremia severa con ingesta de agua menor a la capacidad de excreción renal, porque coexista una secreción aumentada de vasopresina por la misma patología psiquiátrica ${ }^{3,4}$, tabaquismo o fármacos como los neurolépticos, incluido el aripiprazol ${ }^{5,6}$ y los inhibidores de la recaptación de la serotonina ${ }^{7}$. También se reconoce un mecanismo alterado de la sed, con afectación de los núcleos hipotalámicos que rodean al tercer ventrículo, encargados de la regulación de la sed y de la secreción de hormona antidiurética ${ }^{2}$. A nivel molecular la dopamina, que es el neurotransmisor que media el inicio de la ingesta de agua, se ha relacionado con la polidipsia en la psicosis ${ }^{2}$. A estos factores se suma el efecto anticolinérgico de muchos fármacos que aumentan la sensación de sed al provocar sequedad de boca como ocurre con los neurolépticos y antidepresivos tricíclicos ${ }^{1}$.

En nuestro caso existían además otros factores añadidos causantes de hiponatremia, los vómitos en los días previos y el tratamiento de la hipertensión con indapamida. La hiponatremia secundaria a diuréticos es más frecuente en los pacientes tratados con tiazidas, correspondiendo a la indapamida las alteraciones electrolíticas más graves que incluyen también hipopotasemias severas ${ }^{5,8,9}$. El déficit de potasio promueve el paso de éste desde el espacio intracelular al extracelular y esto se equilibra con la entrada de sodio a las células explicando el papel que juega la hipopota- semia en la producción y mantenimiento de la hiponatremia ${ }^{10}$.

Las manifestaciones clínicas de la hiponatremia son fundamentalmente neurológicas y su gravedad depende tanto del valor de la misma como de la rapidez de instauración. La hiponatremia se define como una concentración de sodio en plasma menor de $135 \mathrm{mmol} / \mathrm{L}$. En la hiponatremia aguda, que es aquella que se produce en un plazo inferior a 48 horas, el paso de agua del espacio extracelular al intracelular produce edema de las neuronas con alto riesgo de hipertensión intracraneal y herniación. Si el tiempo de instauración es superior a 48 horas, se trata de hiponatremia crónica ${ }^{5,11}$.

La consecuencia clínica más importante de la hiponatremia es la aparición de encefalopatía por edema cerebral. Los síntomas son variables pudiendo aparecer cefalea, náuseas, vómitos y debilidad; estos síntomas iniciales de la encefalopatía son inespecíficos. Cuando la cifras de sodio en plasma son $<120 \mathrm{mmol} / \mathrm{L}$ o descienden de forma aguda se produce estupor, convulsiones y coma ${ }^{5,11}$.

La insuficiencia respiratoria hipercápnica y el edema pulmonar no cardiogénico son causas de hipoxia en pacientes con hiponatremia sintomática. La hipoxia arterial aumenta el riesgo de daño cerebral porque altera la capacidad cerebral de adaptarse a la hiponatremia. En estos pacientes es necesario mantener una $\mathrm{PO}_{2}>70 \mathrm{mmHg}$ o saturación de oxígeno $>95 \%$ además de corregir la hiponatremia ${ }^{12}$.

El tratamiento de la hiponatremia sintomática aguda constituye una urgencia vital y debe ser independiente de la causa, dado que su mortalidad alcanza cifras de hasta el $15 \%{ }^{11}$. La rapidez de la corrección depende de que haya o no clínica neurológica, del tiempo de instauración y de la magnitud de la hiponatremia. La hiponatremia aguda o grave con clínica neurológica, sobre todo convulsiones y cifras de sodio $<115 \mathrm{mmol} / \mathrm{L}$, debe tratarse con perfusión salina hipertónica al 3\%, mediante bomba de infusión. La velocidad de infusión deberá aumentar la concentración de sodio en el plasma a razón de 1-2 mmol/L durante las primeras 3-4 horas o hasta que cedan las convulsiones y mejore el nivel de conciencia. La reposición debe hacerse con estricto control, midiendo la concentración de electrolitos al 
inicio cada dos horas hasta que el paciente se estabilice neurológicamente. Para evitar un daño cerebral secundario la concentración de sodio en plasma no debería aumentar más de $12 \mathrm{mmol} / \mathrm{L}$ en las primeras 24 horas y $25 \mathrm{mmol} / \mathrm{L}$ durante las primeras 48 horas del tratamiento ${ }^{5,11,13}$.

El paso fundamental previo al tratamiento de una hiponatremia crónica es su diagnostico etiológico, a través de una anamnesis y exploración física detalladas, con evaluación del volumen extracelular y de la volemia arterial circulante eficaz. Las variables de laboratorio útiles en el diagnostico son la osmolalidad del plasma, la osmolalidad urinaria y la concentración de sodio en orina. La respuesta del riñón a la hipoosmolalidad en plasma es la eliminación de una orina cuya osmolalidad es menor de $100 \mathrm{mOsm} / \mathrm{kg}$; esto ocurre en la polidipsia primaria pura. Pero no tenemos que olvidar que en pacientes psiquiátricos muchas veces coexiste con un síndrome de secreción inadecuada de vasopresina que se caracteriza por una orina inapropiadamente concentrada con osmolalidad $>100 \mathrm{mOsm} / \mathrm{kg}^{14}$.

El síndrome de desmielinización osmótica es una complicación del tratamiento de la hiponatremia, que consiste en un cuadro neurológico caracterizado por parálisis flácida, disartria y disfagia. Los factores que influyen son la corrección excesiva y rápida del sodio mayor a $25 \mathrm{mmol} / \mathrm{L}$ en $24-48$ horas, así como la presencia de hipoxia, enfermedad hepática severa o desnutrición sobre todo en relación con el alcoholismo. Sin embargo, se estima que el riesgo de muerte o daño neurológico permanente en pacientes con una hiponatremia severa aguda no tratada es mayor que la posibilidad de lesión desmielinizante tras su corrección. En la clínica no debería plantear dudas el uso de suero salino hipertónico en pacientes sintomáticos con una hiponatremia aguda, pero siempre evitando las correcciones rápidas y excesivas en las primeras 24-48 horas ${ }^{11,13}$.

Por tanto, en los pacientes psiquiátricos como el descrito se combinan diferentes mecanismos en el desarrollo de una hiponatremia grave. El clínico puede disminuir en parte estas alteraciones electrolíticas y sus consecuencias. Es necesaria la monitorización estricta de los iones en los pacientes con patología psiquiátrica siempre que en su tratamiento se asocien determinados fármacos con especial atención a los inhibi- dores de la recaptación de la serotonina, y a los neurolépticos, incluido el aripiprazol, potenciales inductores de una secreción inadecuada de hormona antidiurética. Y por otra parte evitar las tiazidas en el tratamiento de la hipertensión, presente en algunos de estos pacientes.

\section{BIBLIOGRAFÍA}

1. RoBertson GL. Antidiuretic hormone: normal and disordened function. Endocrinol Metab Clin North Am 2001; 30: 671-694.

2. ILLOwSKY BP, KIRCH DG. Polydipsia and hyponatremia in psychiatric patients. Am J Psychiatry 1988; 145: 67-83.

3. Goldman MB, Robertson GL, Luchins DJ, HedeKER D. The influence of polydipsia on water excretion in hyponatremic, polydipsic schizophrenic patients. J Clin Endocrinol Metab 1996; 81: 1465-1470.

4. Spears NM, Leadbetter RA, Shutty MS JR. Clozapine treatment in polydipsia and intermittent hyponatremia. J Clin Psychiatry 1996; 57: 123-128.

5. Adrogue HJ, Madias NE. Hyponatremia. N Engl J Med 2000; 342: 1581-1589.

6. Bachu K, Godkar D, Gasparyan A, Sircar P, Yakoby M, NIRANJAN S. Aripiprazole induced syndrome of inappropriate antidiuretic hormone secretion (SIADH). Am J Ther 2006; 13: 370-372.

7. JACOB S, SPINLER SA. Hyponatremia associated with selective serotonina-reuptake inhibitors in older adults. Ann Pharmacother 2006; 40: 1618-1622.

8. Chapman MD, Hanrahan R, McEwen J, Marley JE. Hyponatraemia and hypokalaemia due to indapamida. Med J Aust 2002; 176: 219-221.

9. Greenberg A. Diuretic complications. Am J Med Sci 2000; 319: 10-24.

10. NGUYen MK, KURTZ I. Role of potassium in hypokalemia-induced hyponatremia: lessons learned from the Edelman equation. Clin Exp Nephrol 2004; 8: 98-102.

11. Fraser CL, AriefF AI. Epidemiology, pathophysiology and management of hyponatremic encephalothy. Am J Med 1997; 102: 67-77.

12. Ayus JC, AriefF AI. Pulmonary complications of hyponatremic encephalothy: non cardiogénico pulmonary edema and hipercapnic respiratory failure. Chest 1995; 107: 517-521.

13. Decaux G, Soupart A. Treatment of symptomatic hyponatremia. Am J Med Sci 2003; 326: 25-30.

14. Singer GG, Brenner MB. Alteraciones de líquidos y electrólitos. En: Kasper DL, Braunwald E, Fauci AS, Hauser SL, Longo DL, Jameson JL, editors. Harrison Principios de Medicina Interna. 16th ed. México: McGraw-Hill Interamericana; 2006: 285-297. 